\title{
PAPR reduction for real baseband OFDM signals
}

\author{
Guangyue Lu, Ping Wu and Catharina Carlemalm-Logothetis
}

Signals and Systems Division, Dept. of Engineering Sciences, Uppsala University

\begin{abstract}
Application of partial transmit sequences (PTS) method for the reduction of peak-to-average power ratio (PAPR) in real-valued OFDM systems is considered in this paper. When directly applying the existing PTS schemes (established based on complex-valued systems) to realvalued systems, one may encounter a difficulty in constructing phase sequences sets that are used to generate the candidates to be chosen for transmission. To overcome the difficulty, the approaches are proposed for two PTS schemes, the adjacent partition (AP-PTS) and the interleaved partition (IP-PTS), to be able to apply to real-valued systems. For the AP-PTS, the difficulty, i.e., the requirement of conjugate symmetry on the phase sequences, is circumvented by re-arranging the data sequence. For the IP-PTS, direct constructing phase sequences sets is avoided; instead the real-valued time domain sequences sets are constructed that correspond to the IDFT of the phase sequences sets, and then used to directly generate the real-valued OFDM candidate signals in the time domain. A distinct feature of the IP-PTS is that only one IDFT is necessary to generate the candidate OFDM signals. Simulations are carried out to evaluate the performance of the AP-PTS and IP-PTS schemes. The results show that both schemes can effectively reduce the PAPR of a real-valued OFDM system.
\end{abstract}

\section{INTRODUCTION}

Orthogonal frequency division multiplexing (OFDM) is a promising technique for high-speed transmission systems owing to its high spectrum efficiency and channel robustness. OFDM baseband signals can be either complex-valued such as those mostly used in wireless systems, or real-valued like discrete multitone (DMT) systems used in ADSL and VDSL [1], and multiband OFDM (MB-OFDM) [2] in Ultra WideBand (UWB) systems. However, large peak-to-average power ratio (PAPR) of transmit signals is one of the main disadvantages of OFDM systems [3]-[13] because it results in low power efficiency and possible nonlinear distortion. It is shown that the PAPR of real-valued baseband OFDM signals is more deteriorative than that of complex-valued baseband OFDM signals [4] .Thus, it is highly desirable to reduce the PAPR of the OFDM signals.

To reduce PAPR, very extensive research has been carried out during the last decade, and various methods have been developed. A review of the methods and techniques for PAPR reduction is given in a recent paper by Han and Lee [3]. Most of the existing methods, e.g., selected mapping (SLM) [5],[6], partial transmit sequences (PTS) [7], [8], [9], that have been developed until now are based on complexvalued OFDM systems. For real-valued OFDM systems, a few methods for PAPR reduction have also been proposed, such as amplitude clipping and filtering [1],[10], a SLM-like method [11], tone reservation [1],[12], etc. Tone reservation method is thought of as to be well suited for DMT ADSL over twisted pair copper because there are several subcarriers which have too low SNR for sending any information and are available for PAPR reduction. But a drawback of this method is its need for a large number of iterations to approach optimal performance.

PTS is one of the most promising and extensively studied methods for PAPR reduction in OFDM. Two main steps in the method are (i) the partitioning of original OFDM signal into a set of sub-blocks and (ii) the adding of the phase rotated subblocks for creating a set of candidate signals from which to choose the one with smallest PAPR for transmission. The PTS can also be expressed as the product of the original OFDM signal with a set of phase sequences, which will be substantiated in Section II. By designing different partitioning schemes or different types of phase sequences, a few PTS schemes have been proposed. When we try to apply the existing PTS schemes to a real-valued system, we notice that it is not an easy thing in designing phase sequences that have to be conjugate symmetric due to OFDM signals being real-valued. However, to the best of our knowledge, little research has been done on the application of the PTS to realvalued OFDM systems. Thus we are motivated to conduct the present work. Finding the approaches to phase sequences problem so as to apply the PTS method to real-valued OFDM systems to reduce their PAPR is the aim of this work.

The rest of this paper is organized as follows: after reformulating a general case of PTS in Section II, the approaches to the phase sequences problem are developed in Section III for two PTS schemes, the adjacent partitioning PTS (AP-PTS) and the interleaved partitioning PTS (IP-PTS), for the PAPR reduction in real-valued OFDM. In section IV, the performance of AP-PTS and IP-PTS is simulated, and conclusions are given in Section V.

\section{GENERAL CASE OF PTS}

A general way of establishing a method to reduce PAPR in an OFDM system is to create a set of candidate time domain (TD) sequences that bear the same information in a transmit signal but have different PAPRs, and then to select from the candidates the one with the smallest PAPR for transmission. The approach of developing PTS with different partitioning schemes is just a special case. This is to be discussed in this section.

Assume $\boldsymbol{X}=\left\{X_{k}\right\}(k=0, \cdots N-1)$ is the frequency domain (FD) data of an OFDM signal $x_{n}=\operatorname{IDFT}\left\{X_{k}\right\}$ 
( $n=0, \cdots, N-1$ ), where $N$ is the number of subcarriers. To reduce the PAPR of signal $\boldsymbol{x}=\left\{x_{n}\right\}$, one generates $M$ candidates (time sequences) as follows,

(1) create $M$ FD data sequences, $\boldsymbol{X}^{\zeta}(\zeta=1, \cdots, M)$, by multiplying $X_{k}$ elementwise with phase sequences $\boldsymbol{P}^{\zeta}=$ $\left\{P_{k}^{\zeta}\right\}(k=0,1, \ldots, N-1)$, respectively, in the following way $\boldsymbol{X}^{\zeta}=\left[P_{0}^{\zeta} X_{0}, P_{1}^{\zeta} X_{1}, \cdots, P_{N-1}^{\zeta} X_{N-1}\right], \zeta=1, \cdots, M$

where $P_{k}^{\zeta}=\exp \left(j \phi_{k}^{\zeta}\right), \phi_{k}^{\zeta}$ is uniformly distributed in $[0,2 \pi)$;

(2) get $M$ TD candidates via IDFTs,

$$
\boldsymbol{x}^{\zeta}=\operatorname{IDFT}\left\{\boldsymbol{X}^{\zeta}\right\}, \quad \zeta=1, \cdots, M
$$

that all bear the same information $x_{n}$ but have different PAPRs. Among $\boldsymbol{x}^{\zeta}$, the one with the smallest PAPR is selected for transmission.

Because phase sequences $\boldsymbol{P}^{\zeta}=\left\{P_{k}^{\zeta}\right\}$ can be created in many different ways, one may have a variety of methods to create $M$ TD candidates $\boldsymbol{x}^{\zeta}$. All existing PTS schemes [8],[9] are considered as special cases of this general idea.

In this section both AP-PTS and IP-PTS are discussed since they will be adopted for real-valued OFDM systems.

\subsection{PTS with adjacent partitions (AP-PTS) [7]-[9]}

The phase sequences $\boldsymbol{P}^{\zeta}=\left\{P_{k}^{\zeta}\right\}$ for AP-PTS can be arranged in the following way,

$$
\boldsymbol{P}^{\zeta}=\left[\boldsymbol{B}_{1}^{\zeta}, \boldsymbol{B}_{2}^{\zeta}, \cdots, \boldsymbol{B}_{V}^{\zeta}\right]
$$

where $\boldsymbol{B}_{v}^{\zeta}=[\underbrace{B_{v}^{\zeta}, \ldots, B_{v}^{\zeta}}_{N / V}], V$ is such an integer that $L=N / V$ is also an integer, and $P_{0}^{\zeta}=\ldots=P_{L-1}^{\zeta}=B_{1}^{\zeta}, P_{L}^{\zeta}=\ldots=P_{2 L-1}^{\zeta}$ $=B_{2}^{\zeta}, \ldots$. In this case, $V$ corresponds to the number of groups into which the sub-carriers are divided, and $\boldsymbol{L}$ is the length of each group. In this scheme, $B_{1}^{\zeta}=1$ is set and $B_{v}^{\zeta}, v=2, \ldots, V$ are selected from the four values $\{ \pm j, \pm 1\}$, so that one may have $M=4^{V-1}$ different phase sequences $\boldsymbol{P}^{\zeta}, \zeta=1, \cdots, M$.

2.2 PTS with interleaved partitions (IP-PTS) [7]-[9]

For IP-PTS method, the phase sequences can be expressed as

$$
\boldsymbol{P}^{\zeta}=[\underbrace{\boldsymbol{D}^{\zeta}, \boldsymbol{D}^{\zeta}, \cdots, \boldsymbol{D}^{\zeta}}_{N / V}]
$$

where $\boldsymbol{D}^{\zeta}=\left[B_{1}^{\zeta}, B_{2}^{\zeta}, \cdots, B_{V}^{\zeta}\right]$ is a phase vector of length $V$ and repeated $L=N / V$ times so that $\boldsymbol{P}^{\zeta}$ has a periodic structure with a period of $V$, i.e., $P_{v+l V-1}^{\zeta}=B_{v}^{\zeta}, v=1, \cdots, V ; l=$ $0,1, \ldots, L-1$. Selecting $B_{v}^{\zeta}$ from a finite number of phase values, one can easily create $M$ phase sequences $\boldsymbol{P}^{\zeta}$.

After $\boldsymbol{X}$ is adjacently or interleavedly partitioned into $V$ subblocks $\boldsymbol{X}^{(v)}$, the $\zeta^{\text {th }}$ candidate signal in AP-PTS or IP-PTS can be written as the weighted sum of partial transmit sequences $\boldsymbol{x}^{(v)}$ [7]-[9], i.e.,

$$
\boldsymbol{x}^{\zeta}=I D F T\left\{\boldsymbol{P}^{\zeta} \boldsymbol{X}\right\}=\operatorname{IDFT}\left[\sum_{v=1}^{V} B_{v}^{\zeta} \boldsymbol{X}^{(v)}\right]=\sum_{v=1}^{V} B_{v}^{\zeta} \boldsymbol{x}^{(v)}
$$

where $\boldsymbol{x}^{(v)}$ is IDFT of $\boldsymbol{X}^{(v)}$.

\section{PTS METHOD FOR REAL-VALUED OFDM SYSTEMS}

For a real-valued OFDM signal $\boldsymbol{x}$, its FD form $\boldsymbol{X}$ is conjugate symmetric, $X_{k}=X_{N-k}^{*}$, where * denotes conjugate. The $M$ candidates $\boldsymbol{x}^{\zeta}$ created for getting one small PAPR OFDM signal for transmission must also be real-valued and their spectra $\boldsymbol{X}^{\zeta}$ must all be conjugate symmetric, $X_{k}^{\zeta}=\left(X_{N-k}^{\zeta}\right)^{*}$. To assure that $X_{k}^{\zeta}=\left(X_{N-k}^{\zeta}\right)^{*}$ are satisfied, the phase sequences $P_{k}^{\zeta}$ must also be conjugate symmetric $P_{k}^{\zeta}=\left(P_{N-k}^{\zeta}\right)^{*}$. However, it is not so easy to find such conjugate symmetric phase sequences with the structure as in (2) for AP-PTS and with the periodic structure as in (3) for IP-PTS. Construction of phase sequences $P_{k}^{\zeta}$ will be the key issue for both AP-PTS and IPPTS to be applied to real valued OFDM systems.

\subsection{AP-PTS for real-valued OFDM systems}

To use AP-PTS for PAPR reduction in a real-valued OFDM system, one can create a new sequence $Y_{k}$ from $X_{k}$ as follows

$$
Y_{k}= \begin{cases}X_{0} / 2, & k=0 \\ X_{k}, & k=1, \cdots, N / 2-1 \\ X_{N / 2} / 2, & k=N / 2 \\ 0, & k=N / 2+1, \cdots, N-1\end{cases}
$$

Since $X_{k}=X_{N-k}^{*}$, then one may have

$$
\begin{aligned}
& (1 / N) \sum_{k=0}^{N-1} X_{k} e^{j \frac{2 \pi}{N} k n}= \\
& (1 / N) \sum_{k=0}^{N / 2} Y_{k} e^{j \frac{2 \pi}{N} k n}+\left[(1 / N) \sum_{k=0}^{N / 2} Y_{k} e^{j \frac{2 \pi}{N} k n}\right]^{*}
\end{aligned}
$$

that is equivalent to

$$
x_{n}^{\zeta}=\operatorname{IDFT}\left\{X_{k}^{\zeta}\right\}=\operatorname{IDFT}\left\{P_{k}^{\zeta} Y_{k}\right\}+\left[\operatorname{IDFT}\left\{P_{k}^{\zeta} Y_{k}\right\}\right]^{*},
$$

which leads to

$$
x_{n}^{\zeta}=2 \operatorname{Re}\left\{I D F T\left\{P_{k}^{\zeta} Y_{k}\right\}\right\}
$$

where $\operatorname{Re}\{a\}$ is the real part of $a, y_{n}^{\zeta}=\operatorname{IDFT}\left\{P_{k}^{\zeta} Y_{k}\right\}$.

shows that the candidates $x_{n}^{\zeta}$ can be obtained from the real part of $y_{n}^{\zeta}$.

Since $x_{n}^{\zeta}=2 \operatorname{Re}\left\{y_{n}^{\zeta}\right\}$, then reducing the PAPR of a realvalued OFDM signal is attainable by reducing the PAPR of 
$\operatorname{Re}\left\{y_{n}^{\zeta}\right\}$. Because the last $(N / 2-1)$ elements in $\boldsymbol{Y}$ are zeros, then the phase sequences are not necessary to be conjugate symmetric when the AP-PTS is used to reduce the PAPR of $\operatorname{Re}\left\{y_{n}^{\zeta}\right\}$, that is, there is no requirement of conjugate symmetry on $P_{k}^{\zeta}$.

However, $Y_{k}$ may have $(N / 2+1)$ non-zero elements. The $(V / 2+1)$ st subblock contains only one element, $Y_{N / 2}$, when the adjacent partitioning scheme is used to partition $Y_{k}$ into $V$ subblocks. To reduce the complexity of the algorithm, element $Y_{N / 2}$ is moved to the $(V / 2)^{\text {th }}$ subblock so that it contains $N / V+1$ elements of the form

$$
\boldsymbol{Y}^{(V / 2)}=\left[0, \cdots, 0, Y_{\left(\frac{v}{2}-1\right) \frac{N}{V}}, Y_{\left(\frac{\nu}{2}-1\right) \frac{N}{V}+1}, \cdots, Y_{\frac{N}{2}-1}, Y_{\frac{N}{2}}, 0, \cdots, 0\right]
$$

And the first $(V / 2-1)$ subblocks $\boldsymbol{Y}^{(v)}(v=1, \ldots, V / 2-1)$ are generated using the conventional way [8], [9]. The remaining $V / 2$ subblocks $\boldsymbol{Y}^{(v)}(v=V / 2+1, \ldots, V)$ all are zeroes.

Since, in practical systems, such as, ADSL [1] and MBOFDM [2], the $(V / 2)^{\text {th }}$ subcarrier is not used for data transmission (in other words $X_{N / 2}=Y_{N / 2}=0$ ), all of the subblocks $\boldsymbol{Y}^{(v)}(v=1, \ldots, V / 2)$ can be obtained by the conventional adjacent partitioning scheme.

After re-formulating the original OFDM data sequence $X_{k}$ in a new form $Y_{k}$ in (5), the conventional AP-PTS method can be applied to a real-valued OFDM system. Mathematically, it can written as,

$$
\begin{aligned}
\left\{B_{1}, B_{2}, \cdots, B_{\frac{v}{2}}\right\} & =\arg \min _{B_{1}^{\zeta}, \cdots, B_{v / 2}^{\zeta}} \operatorname{PAPR}\left\{x_{n}^{\zeta}\right\} \\
& =\arg \min _{B_{1}^{\zeta}, \cdots, B_{v / 2}^{\zeta}} P A P R\left\{\operatorname{Re}\left(y_{n}^{\zeta}\right)\right\}
\end{aligned}
$$

where $y_{n}^{\zeta}=\sum_{v=1}^{V / 2} B_{V}^{\zeta} \operatorname{IDFT}\left\{Y^{(v)}\right\}$.

Because the first $V / 2$ subblocks in a new data sequence $Y_{k}$ are non-zero, $4^{V / 2-1}$ different phase sequences can be obtained in such situation and only the $V / 2$ phase factors, $B_{1}, B_{2}, \cdots, B_{V / 2}$, in each phase sequence are needed. And the $V / 2$ phase factors resulting in the lowest PAPR are needed to transmit as side information to the receiver.

\subsection{IP-PTS for real-valued OFDM systems}

As shown in (3), the phase sequences for IP-PTS have a periodic structure with a period of $V$. Due to the periodic structure in the FD, the IDFT of $P_{k}^{\zeta}$ in (3) may be expressed as [13],

$$
\begin{aligned}
p_{n}^{\zeta} & =\operatorname{IDFT}\left[P_{k}^{\zeta}\right]= \begin{cases}d_{n / L}^{\zeta}, & n=0, L, 2 L, \cdots,(V-1) L \\
0, & \text { otherwise }\end{cases} \\
& =\sum_{i=0}^{V-1} d_{i}^{\zeta} \delta(n-i L), \quad n=0, \cdots, N-1
\end{aligned}
$$

where $L=N / V, \boldsymbol{d}^{\zeta}=\operatorname{IDFT}\left[\boldsymbol{D}^{\zeta}\right]$. Then,

$$
x_{n}^{\zeta}=I D F T\left\{P_{k}^{\zeta} X_{k}\right\}=x_{n}^{*} p_{k}^{\zeta}
$$

$$
\begin{aligned}
& =\sum_{i=0}^{V-1} d_{i}^{\zeta}\left[x_{n} * \delta(n-i L)\right] \\
& =\sum_{i=0}^{V-1} d_{i}^{\zeta} x_{\left((n-i L)_{N}\right)} R_{n}
\end{aligned}
$$

where * denotes the circular convolution, $\left((n)_{N}\right)$ denotes $n$ modulo $N$ and $R_{n}$ is the rectangular sequence which is defined as $R_{n}=\left\{\begin{array}{l}1,0 \leq n \leq N-1 \\ 0 \text {, otherwise }\end{array} \cdot x_{\left((n-i L)_{N}\right)} R_{n}\right.$ is the circular shift of $x_{n}$. From (11), one can see that, to ensure $x_{n}^{\zeta}$ to be real-valued (which is required by a real-valued OFDM system), $d_{i}^{\zeta}$ must be real-valued noting that both $x_{n}$ and $R_{n}$ are real-valued. Instead of direct finding independent phase sequences sets $\boldsymbol{P}^{\zeta}$, we work on TD sequence $d_{i}^{\zeta}$.

A simple, feasible scheme is that $d_{i}^{\zeta}$ take on one of the three real values, $-1,0,1$, and is used in the simulations below in Section VI. It should be pointed out that, when $d_{i}^{\zeta}$ $=0$, the corresponding circularly shifted sequence is not includes in (11) so that the computational complexity can be reduced. To recover the transmitted data at the receiver, $\boldsymbol{D}^{\zeta}$ should not contain any zero elements. To ensure that $\boldsymbol{D}^{\zeta}$ has no zero elements, the offline exhaustive searching of $\boldsymbol{D}^{\zeta}=$ $\operatorname{DFT}\left\{\boldsymbol{d}^{\zeta}\right\}$ is first performed. All the independent sequences $\boldsymbol{d}^{\zeta}$ whose frequency-domain data $\boldsymbol{D}^{\zeta}$ have non-zero elements are selected out and put in a sequence set $Q$ from which to choose $M$ sequences to build $M$ OFDM candidate signals according to (11). Hence, the IP-PTS for real-valued OFDM signals can be written as,

$$
\left\{d_{0}, d_{1}, \cdots, d_{V-1}\right\}=\arg \min _{d^{\zeta} \in Q} P A P R\left\{\sum_{i=0}^{V-1} d_{i}^{\zeta} x_{\left((n-i L)_{N}\right)} R_{n}\right\}
$$

Because a large number of sequences $\boldsymbol{d}^{\zeta}$ are available when $V$ is sufficiently large (e.g., $V=8$ ), there is no difficulty in getting enough different sequences $\boldsymbol{d}^{\zeta}$ from set $Q$. It is easily found that, since $d_{i}^{\zeta}$ are real-valued, the phase sequences $\boldsymbol{P}^{\zeta}$ will be conjugate symmetric and thus $\boldsymbol{X}^{\zeta}=$ $\boldsymbol{X} \boldsymbol{P}^{\zeta}$ will also be conjugate symmetric.

\section{SIMULATIONS, RESULTS AND CONCLUSIONS}

The performance of the proposed PTS schemes for realvalued OFDM systems is evaluated by means of computer simulations. All the simulations are carried out based on 11000 independent real-valued QPSK-modulated OFDM symbols if not stated otherwise. Comparisons are made from the simulated results for the proposed PTS schemes, SLM method and normal OFDM. For SLM, $M_{1}$ independent phase sequences with conjugate symmetry are generated in each simulation. 
To evaluate the performance, the complementary cumulative distribution function (CCDF) of PAPR for OFDM signal $x$, that is,

$$
C C D F(P A P R(x))=\operatorname{Prob}(P A P R(x)>P A P R 0)
$$

is calculated. CCDF can be interpreted as the probability that the PAPR of an OFDM signal exceeds some clip level PAPRO (which is referred to as the symbol clip probability and determined by the amplifiers in the system).

Comparison of the performance of AP-PTS with $V=8$, IPPTS with $V=8$ and SLM with $M_{1}=4$ for a real-valued OFDM $(N=128)$ is shown Fig. 1 . For IP-PTS, $M=16$ and $M=64$ phase sequences with three zero-elements (i.e., $N_{0}=3$ ) are selected from set $Q$, respectively. Here $N_{0}$ denotes the number of zeroelements in $\boldsymbol{d}^{\zeta}$. In this case, only five circularly shifted sequences in (11) are used. The number of IDFT operations in SLM, AP-PTS and IP-PTS are 4, 4 and 1, respectively. And the number of the candidates in SLM, AP-PTS, IP-PTS $(M=16)$ and IP-PTS $(M=64)$ are 4, 64, 16 and 64, respectively. The comparison in Fig.1 demonstrates that three methods can reduce PAPR effectively. With the same number of IDFT operations, AP-PTS has better performance than SLM. For example, the AP-PTS has about $1.7 \mathrm{~dB}$ gain at $0.1 \%$ probability compared to the SLM. The performance of AP-PTS and IP-PTS is almost similar when the same number of candidates is used in AP-PTS and IP-PTS $(M=64)$. However, only one IDFT is needed in the IP-PTS, while $V / 2$ IDFTs are needed in the AP-PTS, which indicates that the IPPTS has lower computational complexities than the AP-PTS. The comparison of IP-PTS $(M=16)$ and IP-PTS $(M=64)$ shows that the performance of IP-PTS is improved with the increase of the number of candidates.

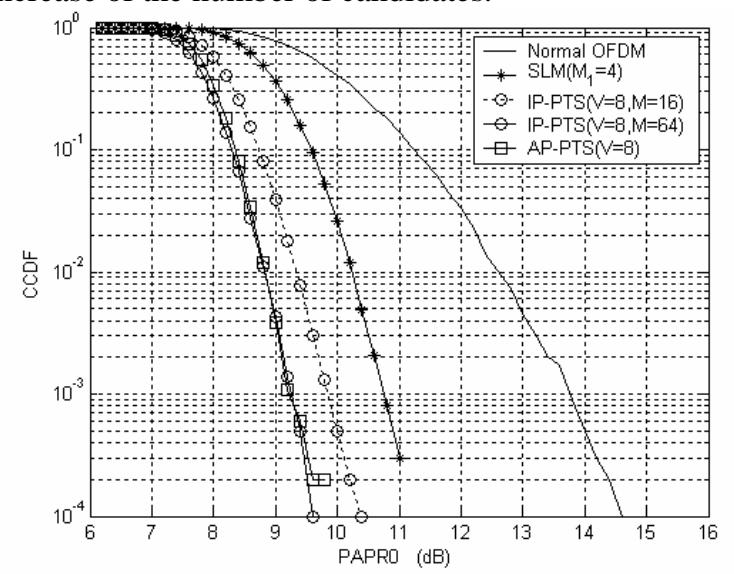

Fig.1 Comparison of AP-PTS, IP-PTS and SLM $(N=128)$

The variation of AP-PTS' and IP-PTS' performance with the number of subblocks is also investigated. The MBOFDM [2] data model is used in the investigation. The simulated results are shown in Fig.2. The data in both APPTS and IP-PTS are all partitioned into 4 and 8 subblocks, respectively. For IP-PTS with $V=4,4$ phase sequences are selected from $Q$ in simulation in order that IP-PTS has the same candidates as AP-PTS ( $V=4)$. From Fig.2, it can be seen that the PAPR of MB-OFDM is reduced with both AP-PTS and IP-PTS. The performance of both methods can be improved with increase the number of subblocks, for example, at $\mathrm{CCDF}=0.01 \%$, the PAPR0 for IP-PTS $(V=4)$ and IP-PTS $(V=8)$ are $12.8 \mathrm{~dB}$ and $9.5 \mathrm{~dB}$, respectively, compared to $14.3 \mathrm{~dB}$ for the normal OFDM.

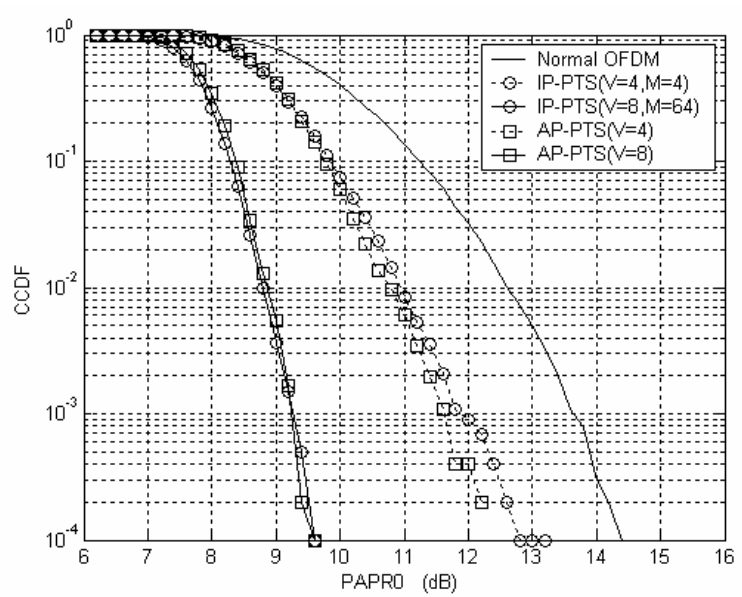

Fig.2 Performance variation of AP-PTS and IP-PTS with the number of subblocks for MB-OFDM signals

The similar simulations as in Fig.2 are also carried out for a DMT ADSL signal [1],[12], where 64-QAM modulation is employed to generate the baseband data. There are 512 subcarriers, and the tones 33-256 are user for the data transmission. The simulation results are shown in Fig.3. The target symbol clip probability in DMT ADSL signals could be chosen as $5 \times 10^{-5}$ [1],[12], which is the minimum of the vertical axis in Fig.3. From Fig.3, one can see that both the AP-PTS and the IP-PTS can lower the PAPR of the DMT ADSL signals greatly. The two algorithms have almost same performance for 64 candidates; and the performance of the AP-PTS is slightly better than that of the IP-PTS when 4 candidates are used in both methods. And the performance of both the methods is increased with the increase of the number of subblocks. Conclusions that both the AP-PTS and the IP-PTS are feasible for DMT ADSL and MB-OFDM systems, which are real-valued, can be drawn from Fig.2 and Fig.3.

Because, in the IP-PTS, different sequences $\boldsymbol{d}^{\zeta}$ can be selected from set $Q$ for simulations, Fig. 4 gives the performance of IP-PTS when we select 16 phase sequences with $N_{0}=1, N_{0}=3$ and $N_{0}=5$, respectively. It shows that there is no great difference among these three situations. Clearly, the more zero-elements the phase sequences have, the less computational complexity the IP-PTS needs. However, no many phase sequences are available when $N_{0}=5$. 


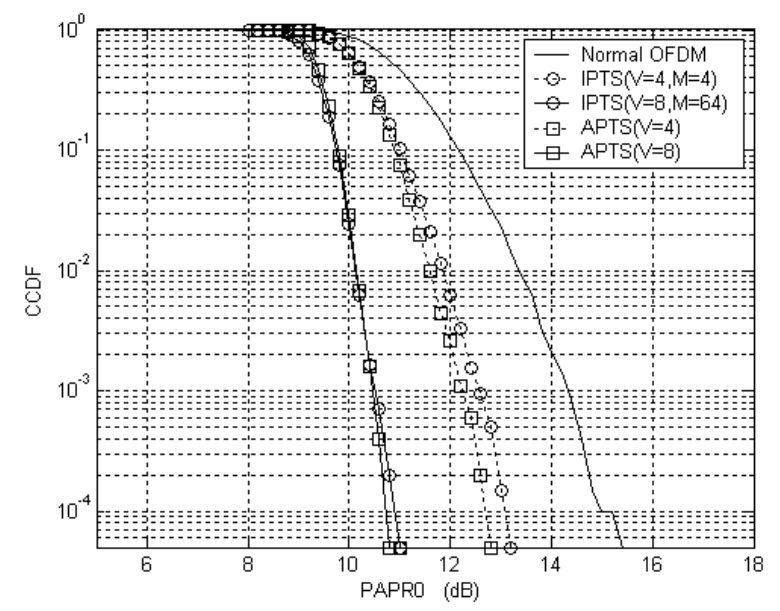

Fig.3 Performance variation of AP-PTS and IP-PTS with the number of subblocks for DMT ADSL signals

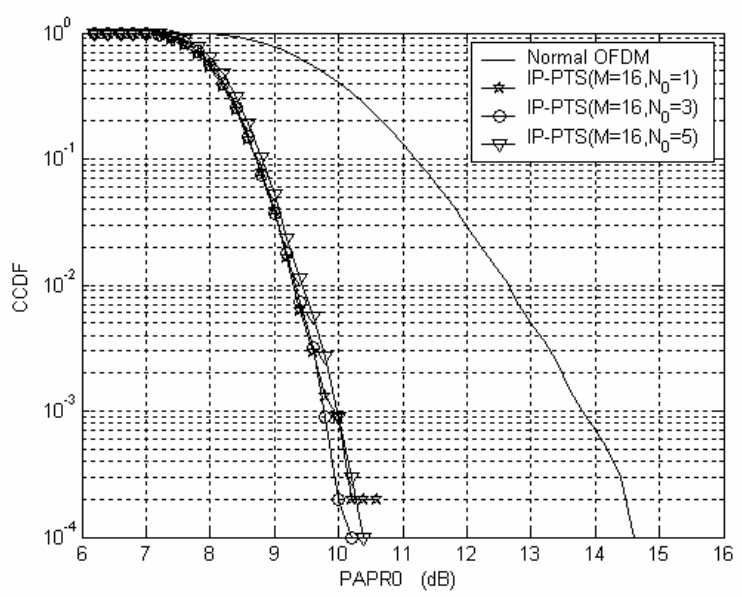

Fig.4 IP-PTS performance with phase sequences with $N_{0}=1$, $N_{0}=3$ and $N_{0}=5$

\section{SIMULATIONS, RESULTS AND CONCLUSIONS}

The PTS method in two different schemes, the AP-PTS and the IP-PTS, has been extended to reduce the PAPR of real-valued OFDM signals. The approaches to the key problem with the construction of phase sequences (used for constructing real-valued OFDM candidate signals) that must be conjugate symmetric have been proposed for the two PTS schemes.

For the AP-PTS, the data sequences are re-arranged in such a way that the requirement of conjugate symmetry on the phase sequences is released, namely, the constructed phase sequences are not necessarily conjugate symmetric, which makes the problem be easily solved. For the IP-PTS, the real-valued TD sequences sets corresponding to the IDFT of the phase sequences sets (instead of the phase sequences sets themselves) are constructed, and then used to create the real-valued OFDM candidate signals in the time domain.

The results show that the extension is successful and the proposed AP-PTS and IP-PTS for real-valued OFDM systems can effectively reduce PAPR. The performance of AP-PTS and IP-PTS is improved with the increase of the number of subblocks. For the same number of candidates, AP-PTS has similar performance as IP-PTS, but the AP-PTS needs $V / 2$ IFFT operations while the IP-PTS needs only one. Thus, the latter method has lower computational complexity.

Acknowledgement: This work was supported partly by Scholarship from China Scholarship Council, China National Key Lab of Space Microwave Technology (No. 51473020203QT6702), and partly by NEWCOM (Network of Excellence in Wireless COMmunications) under the sixth Framework Program of European Unioin.

\section{REFERENCES}

[1] ITU-T, Asymmetric digital subscriber line (ADSL) transceivers, Recommendation G.992.1, June, 1999.

[2] A. Batra et.al, "Multi-band OFDM physical layer proposal for IEEE 802.15 Task Group 3a”. Sep.14, 2004.

[3] S. H. Han and J. H. Lee, "An overview of peak-toaverage power ratio reduction techniques for multicarrier transmission”, IEEE wireless commun., 12(2), pp.56-65, Apr., 2005.

[4] H. Yu,M. Chen and G. Wei, "Distribution of PAR in DMT systems”, Electron. Lett.,39, pp. 799-801, May 2003.

[5] R.W. Bauml, R.F.H. Fisher and J.B. Huber, "Reducing the peak-to-average power ration of multicarrier modulation by selected mapping”, Electron. Lett., 32, pp.2056-2057, Oct., 1996.

[6] M.Breiling, S. H. Muller, and J.B. Huber, "SLM peakpower reduction without explicit side information”, IEEE Commun. Lett., 5(6),pp.239-241,June 2001.

[7] S.H.Muller, and J.B. Huber, "OFDM with Reduced Peak to Average Power Ratio by Optimum Combination of Partial Transmit Sequences”, Electron. Lett., 33, pp.368369, Feb., 1997.

[8] S.G. Kang, J.G. Kim., and E. K. Joo, “A Novel Subblock Partition Scheme for Partial Transmit Sequence OFDM”, IEEE Trans. Broadcasting,45(3),pp.333-338, Sept. 1999.

[9] S.H. Muller, and J.B. Huber, "A novel peak power reduction scheme fot OFDM”,Proc. IEEE PIMRC'97, Helsinki, Finland,Sept.1997, pp. 1090-1094.

[10] A. Gatherer, and M. Polley, "Controlling clipping probability in DMT transmission”, in Proc. Asilomar Conf. on Signals, systems, and computer, vol.1,pp.578584, Pacific Grove,Calif., USA, Nov. 1997

[11] D. J. G. Mcstdagh and P.M.P. Spruyt, "A method to reduce the probability of clipping in DMT-based transceivers”, IEEE Trans. Commun., 44(10), pp. 12341238, Oct.,1996.

[12] N. Petersson, "Peak and power reduction in multicarrier systems”, Licentiate thesis, Lund University, Lund, Sweden, Nov. 2002.

[13] G. Lu and P. Wu, "PAPR reduction in OFDM based on weighted sum of cyclically shifted sequences", Proc. of 10th International OFDM-Workshop (InOWo'05), Aug.31-Sept.1, 2005, Humburg, Germany, pp.210-214. 